\title{
Using Serious Games to Train Adaptive Emotional Regulation Strategies
}

\author{
Mariano Alcañiz ${ }^{1,2}$, Alejandro Rodríguez ${ }^{1}$, Beatriz Rey ${ }^{1,2}$, and Elena Parra ${ }^{1}$ \\ ${ }^{1}$ Instituto Interuniversitario de Investigación en Bioingeniería y Tecnología Orientada al Ser \\ Humano, Universitat Politècnica de València, I3BH/LabHuman, Camino de Vera s/n, 46022 \\ Valencia, España \\ ${ }^{2}$ Ciber, Fisiopatología de Obesidad y Nutrición, CB06/03 Instituto de Salud Carlos III, Spain \\ \{arodriguez, brey, malcañiz\} @labhuman.i3bh.es
}

\begin{abstract}
Emotional Regulation (ER) strategies allow people to influence the emotions they feel, when they feel them, how they experience them, and how they express them in any situation. Deficiencies or deficits in ER strategies during the adolescence may become mental health problems in the future. The aim of this paper is to describe a virtual multiplatform system based on serious games that allows adolescents to train and evaluate their ER strategies. The system includes an ecological momentary assessment (EMA) tool, which allows the therapist to monitor the emotional status of teenagers every day in real time. Results obtained from a usability and effectiveness study about the EMA tool showed that adolescents preferred using the EMA tool than other classical instruments.
\end{abstract}

Keywords: Serious Games, Emotional Regulation, Ecological Momentary Assessment, Virtual Reality.

\section{Introduction}

Emotional Regulation (ER) strategies allow people to influence the emotions they feel, when they feel them, how they experience them, and how they express them in any situation. These strategies may be automatic or controlled, voluntary or involuntary. ER strategies should be differentiated depending on when they have their first impact on the emotion generation process. Despite the fact that there are diverse emotional regulation strategies, most research has been conducted into cognitive reevaluation (assigning a non-emotional meaning to an event) and expressive suppression (controlling the somatic response to an emotion). Both the physical and psychological implications will be different depending on the strategy used.

Numerous studies indicate the role that these regulation strategies play in the development and maintenance of adaptive and healthy behavior. Previous research [1] has concurred in finding diverse emotional regulation dysfunctions in the clinical population with depressive disorders. Deficiencies or deficits in ER are related to the occurrence of numerous mental and physical health problems, including borderline 
personality disorder, depression, anxiety, problems of social interaction, problems of adaptations, violent behavior and other disruptive behaviors.

These deficits become especially evident during the adolescence and may become a psychosocial problem. Actually, schools and high-schools denounce a worrying increase in indiscipline and episodes of physical and psychological violence in the classroom. Proof of this concern is the Study of Violence report by the Centro Reina Sofía or Cisneros X [2-3] which showed that high percentage values of children had suffered bullying or school-based violence.

Many of these disruptive behaviors by the adolescents are built upon deficiencies in situation selection, situation modification, attention deployment, cognitive change or response modulation [4]. On the other hand, an adaptive ER involves choosing and implementing ER strategies that are adapted to the context, adapted to how controllable the internal and external events are, and in harmony with long term goals [1]. Berking believes that the effectiveness of behavior change therapies could be improved by identifying general emotional regulation abilities, developing interventions that could improve these abilities and including these interventions in the therapies [5].

The current tools used to evaluate possible deficits in ER strategies are based on subjective questionnaires, such as the Emotion Regulation Checklist [6] or the Emotion Regulation Questionnaire [7]. These questionnaires ask subjects about how they feel and manipulate their emotions in controlled environments or in common situations in real life. Another way of evaluating the ER strategies used is through tests in laboratory, where the subjects ought to do emotion generating tasks with the aim to analyze the strategies that have to be used [8]. Even though these tools are very useful for therapist (distinguishing between clinical and normal populations), they present some limitations; specifically, they are unattractive for the evaluation in adolescents' population, who are particularly reluctant to be assessed.

These limitations can be overcome with the development of instruments based on new technologies. Specifically, virtual reality (VR) systems and Information and Communication Technologies (ICT) are a technology that are very attractive and very common to young people and adolescents, who are accustomed to the simulated environments of videogames.

The development of new interactive technologies based on VR has already had some influence on certain aspects of the teaching/learning environment. VR and its application to youth education is a relatively young field but interest is growing due to its strong motivational impact [9] and the critical reasons for why and how graphicbased VR can greatly enhance the quality of education provided [10]. The devices and techniques allow navigating and interacting through VR environments in more intuitive and more natural ways. In addition, immersive and persuasive capacities of VR make it a highly appropriate technology for assessment and intervention task.

Recently, several works have used new techniques based on ICT for training of ER strategies. Playmancer project [11] is a system that uses multimodal recognition of emotions, in combination of serious games for the training of ER strategies, with the purpose of treating psychological and behavioural disorders [12]. In other project, Replay [13], a system based on a VR environment with avatars and tracking technologies was developed with the aim of improving the emotional implication of participants (students with behaviour disorders) and training ER strategies. 
In this work, we describe GameTeen, a virtual multiplatform system based on serious games that allows teenagers to train and evaluate their ER strategies. Furthermore, the system includes an ecological momentary assessment (EMA) tool, which allows the therapist to monitor the emotional status of adolescent every day in real time. Finally, the results obtained of a usability and effectiveness study about the EMA tool will be presented.

\section{Methods}

The GameTeen system is a virtual multiplatform system based on serious games designed for teenagers, which allows training, evaluating and monitoring their emotions and the ER strategies that they apply everyday. GameTeen was developed according to recommendations of intervention program principles, which claim that a way of training and assessing how ER strategies have been applied by subjects involves the use of mood induction procedures. In the context of these procedures, subjects can learn to analyze the emotion felt and the ER strategies that they employed to regulate themselves [14].

Following this recommendation, GameTeen system was designed in three blocks. A first block would be a system where a positive or a negative emotion is induced in the subjects; it consists of two Mood Induction Games. A second block would consist of two mini-games where the participants may train two ER strategies (ER Strategies Training). Finally, in the third block, an ecological momentary assessment (EMA) would allow the therapist to monitor the emotional status of adolescent every day in real time.

\subsection{Induction Games}

The aim of the Mood Induction Games is to induce two different emotions in the participants. The emotions induced were joy and frustration. To achieve the desirable mood induction, several tools of positive and negative reinforcement were used respectively, such as music, sounds, emotional messages, etc. The monitoring of emotional levels in the two games was performed through periodic interruptions where the game asked participants to indicate their emotional level on a scale of 0 to 10 represented as thermometer.

Joy Induction Game. In the joy game (Figure1) the action took place in an attraction park. In this game the participants had to exploit as many balloons as possible of a shower of balloons. For this, the participants had several weapons and ammunitions that they were getting through the performance of the different ER strategies. The game had ten levels of difficulty that affected to the number of balloons and the velocity of appearance. 


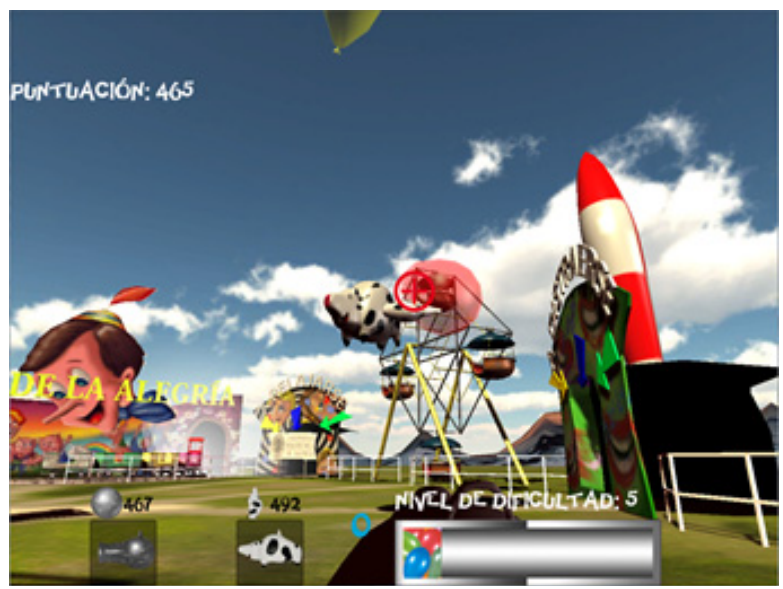

Fig. 1. Joy Induction Game. The game is located in an attraction park scene. In this scene, many balloons appear in continuous way while playing the game.

Frustration Induction Game. In the frustration game (Figure 2), a version of the "whack a mole" game was developed. The game is located in a rural land scene where there are several holes. In these holes, several moles can appear in a continuous way while playing the game. In this game, the participant has to whack all moles, at the same time that they are appearing, with a virtual mace. The game had three levels of difficulty that are related with the velocity and frequency of appearance of the moles. To achieve the frustration induction, the game was programmed so that some hits are not accurate and the participants are penalized.

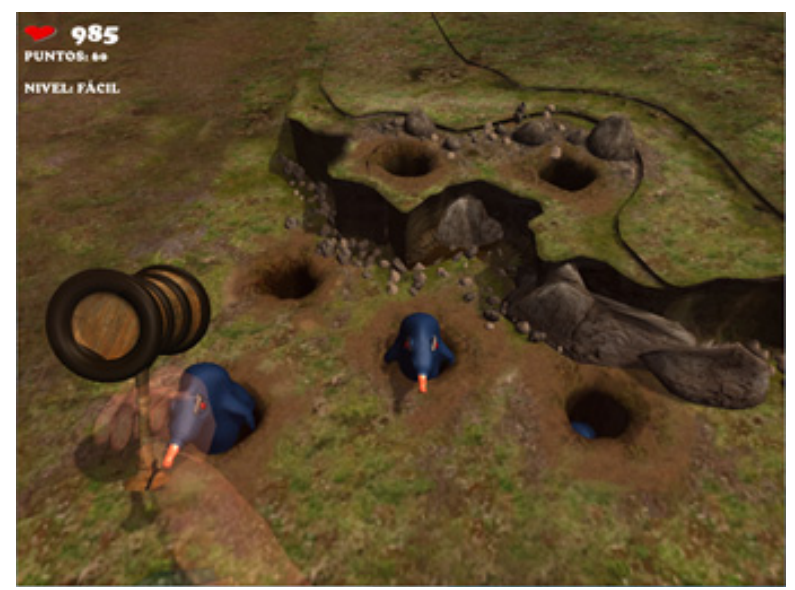

Fig. 2. Frustration Induction Game. The game is located in a rural land scene where there are several holes. In these holes, several moles can appear in a continuous way while playing the game. 


\subsection{ER Strategies Training (Mini-Games)}

Apart from these mood induction games, two mini-games were developed for training ER strategies. One of them consists of a respiration task where the subjects have to breathe at the same rate as indicated in the mini-game. This respiration strategy consists of a feather (Figure 3a), which goes up and down in the screen. In the other one, subjects have to click on all the numbers that appear sequentially on screen except one number, which is indicated within the mini-game (Figure $3 b$ ).
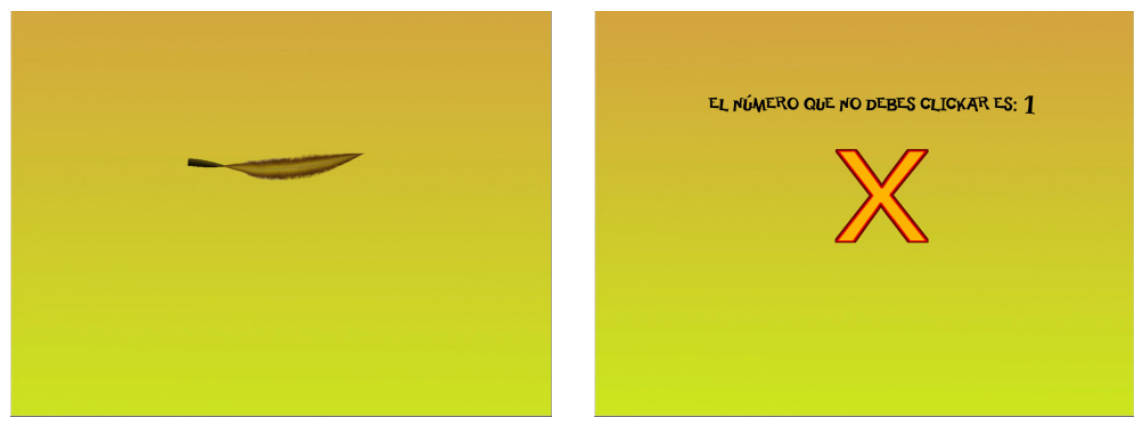

Fig. 3. Emotional Regulation Strategies Training. In the respiration strategy (a) consist in a feather that goes up and down in the screen to velocity moderate; and the distraction strategy (b) consist an sequence of number and one cross that are appearing sequentially.

\subsection{Ecological Momentary Assessment (EMA)}

To evaluate continuous changes in the emotions of subjects in their daily life we also developed an EMA system (Figure 4). The purpose of this system is to have a diary record of the emotions felt by the subjects by means of an android device, which can be configured and monitored from a webpage. This system includes a custom avatar who asks a set of questions about the feeling of the teenagers in predefined moments during the day. Moreover, the system has a web page where the therapist can authenticate himself/herself with the purpose of monitoring the emotional activity of all subjects in his/her study.
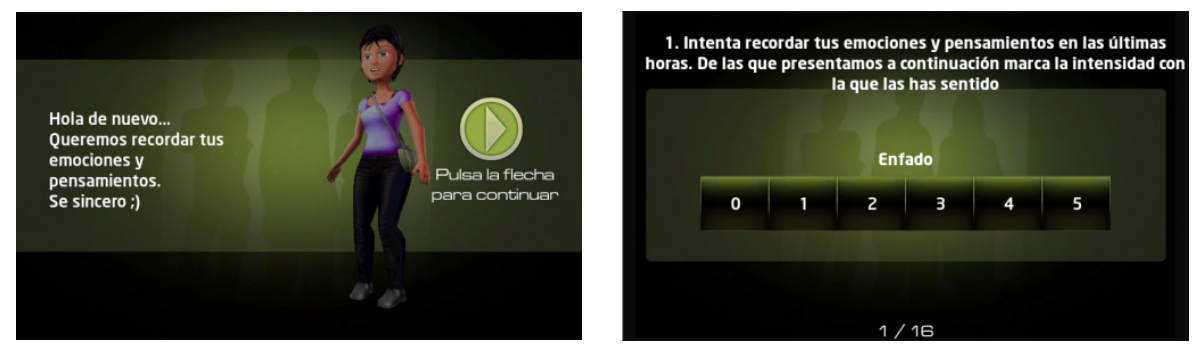

Fig. 4. EMA. Capture an example avatar and the example a question. 


\subsection{Technological Aspects}

The Mood Induction Games and the Mini-Games were developed with the purpose of being used both on PC and on Android platforms (Figure 5). For this reason, we used the Unity3d as the development environment. It was programmed in C-Sharp. CSharp is a friendly programming language that has many communication devices libraries and web services libraries.
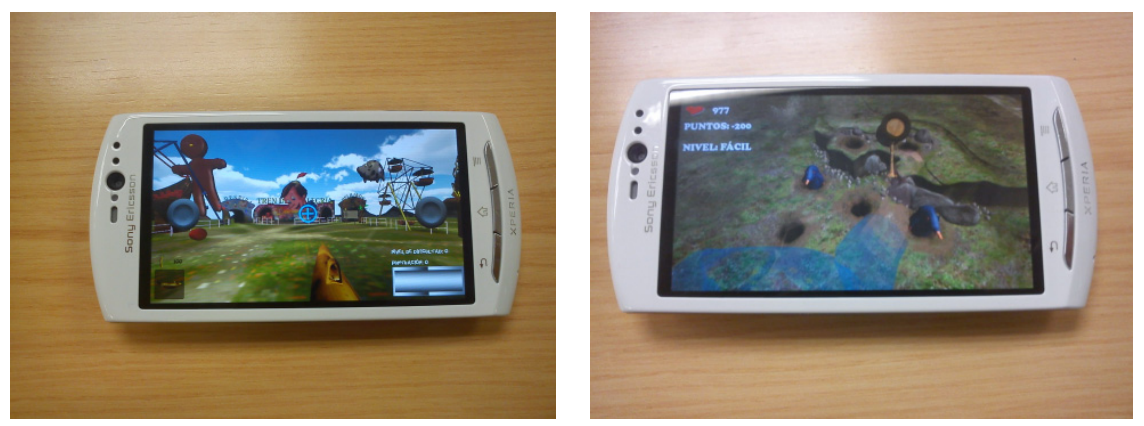

Fig. 5. Induction Games in an Android smartphone

Mainly, the PC platform was thought to be used with keyboard and mouse in the participants' home but in addition this system has the option to be used in laboratory under therapeutic supervision. For this, GameTeen has the option of registering the ECG signal of participants synchronized with game events, allowing therapists to monitor changes in the heart rate in real time in laboratory conditions.

\subsection{Design Usability Study}

The GameTeen platform has been evaluated with students of a high school. In this work, we present the preliminary results obtained of a usability study performed with teenagers.

Twenty one participants, 10 men and 11 women, were evaluated in this study, all of them with ages between 12-14 years old (mean 13.20 years old) with normal o corrected-to-normal vision. The subjects were students of Ramón Llull High-School of València without any psychological disorder and none of them was under psychological supervision. Their parents signed an informed consent for allowing their data to be used in this study.

The adolescents were divided in two groups. The first group had to express their emotions daily through the EMA system. For that, we lent several smartphones (Sony Xperia Neo V), whose functions were banned to except the EMA system. On the other hand, the second group had to do the same task; however they used questionnaires in paper. The questions in paper were the same that the EMA system requested. All participants had to do this task twice a day during one week. At the end of the first week, groups were exchanged, and the participants had to do the same task during other week. 
When all participants were in the two groups, we asked to them that they completed the usability questionnaire, which registered information about the usability of the paper questionnaire, EMA system and the comparative between the two procedures to daily register of emotions.

\section{Results}

In this work, we present preliminary results obtained in the usability of EMA system when this is compared with the usability of classical tools used to measured emotions, questionnaires in paper.

Results showed that teenagers perceived more discomfort while they did the paper questionnaires than with the EMA system $(\mathrm{p}=0.001)$. The $50 \%$ of subjects confirmed that the EMA system was very comfortable and its alarms were useful to remember the teenagers to complete the questions. This was reflected in the fact that the $100 \%$ of subjects perceived as negative quality that the paper questionnaire did not have any alarm. Against, the $19 \%$ of adolescents liked having freedom of schedule to answer the paper questionnaire.

On the other hand, the participants appreciated that the EMA system was more useful than paper questionnaire $(p=0.004)$. The $42 \%$ participants indicated that the EMA system was easier to use than paper questionnaires, and the $100 \%$ participants showed their disagreement by having to move so many papers from one place to another.

\section{Discussion}

This paper describes the GameTeen project, a psychotherapeutic multiplatform based on serious games to train and evaluate the ER strategies in teenagers. This multiplatform was developed using news technologies in VR and ICTs with the aim to improve the classical tools used in the field of ER, to improve the motivation and to help to catch the attention of teenagers that can hardly be attracted by other instruments, such as questionnaires.

GameTeen allows the adolescents to train two ER strategies when a positive or negative emotion has been induced them. These ER strategies are based on two minigames, a slowly respiration task and an attention task. In addition, GameTeen helps the therapist to evaluate the ER strategies used by the subjects and it also enables to follow the emotion felt during the evaluation. For this reason, GameTeen system has been developed for being used as much in a controllable situation through a PC, as day-to-day through an EMA system.

In this paper, we have showed preliminary results obtained of a usability study, which was carried for the EMA system. The obtained results suggest that the EMA system can be used to monitor emotions in the adolescent population.

The participants appreciated the comfortably of EMA above the paper questionnaire. In addition, they emphasized that the EMA system was very useful. This can be caused by different factors: the ease of transportation; the fact that the paper 
questionnaire was a huge number of pieces of paper; and the usefulness of the reminders through the alarms. These qualities were not presented in paper format.

However, we are aware that the population used for this analysis was a non-clinical population. This will require, in the future, to validate the system with a clinical population of teenagers, i.e. with adolescents who have been diagnosed an ER disorder. Nevertheless, because of these results, we are optimistic and we believe that the system might be used to help therapists to monitor the emotions with a clinical population.

In future works, we will evaluate the reliability of GameTeen as therapeutic tool for the early detection of ER patterns with a high risk of leading to health or behavioral problems. In addition, we will analyze how their use as training tool would enable their integration into psychoeducational programs for prevention of emotional disorders. Finally, we will also use physiological measurement tools, such as electroencephalogram, to study the influence of the ER strategies in the human physiologic behavior with the aim of measuring and quantifying this effect.

Acknowledgments. This study was funded by Vicerrectorado de Investigación de la Universitat Politècnica de València, Spain, PAID-06-2011, R.N. 1984; by Ministerio de Educación y Ciencia, Spain, Project Game Teen (TIN2010-20187) and partially by projects Consolider-C (SEJ2006-14301/PSIC), "CIBER of Physiopathology of Obesity and Nutrition, an initiative of ISCIII" and Excellence Research Program PROMETEO (Generalitat Valenciana. Consellería de Educación, 2008-157).

The work of Alejandro Rodríguez was supported by the Spanish MEC under an FPI Grant BES-2011-043316.

\section{References}

1. Mennin, D., Farach, F.: Emotion and evolving treatments for adult psychopathology. Clinical Psychology: Science and Practice 14, 329-352 (2007)

2. Serrano, A., Iborra, I.: Informe violencia entre compañeros en la escuela. Spanish Version (2005), http: / /www. centroreinasofia.es

3. Informe Cisneros X.: Acoso y Violencia Escolar en España, por Iñaki Piñuel y Araceli Oñate. Editorial IIEDDI, Spanish Version (2007)

4. Werner, K., Gross, J.J.: Emotion Regulation and Psychopathology. In: Emotion Regulation and Psychopathology: A Transdiagnostic Approach to Etiology and Treatment. Guildford Press (2010)

5. Berking, M., Wupperman, P., Reichardt, A., Pejic, T., Dippel, A., Znoj, H.: Emotionregulation skills as a treatment target in psychotherapy. Behaviour Research and Therapy $46,1230-1237$ (2008)

6. Shields, A., Cicchetti, D.: Emotion regulation among school-age children: The development and validation of a new criterion Q-sort scale. Developmental Psychology 33(6), 906-916 (1997)

7. Gross, J.J., John, O.P.: Individual differences in two emotion regulation processes: Implications for affect, relationships, and well-being. Journal of Personality and Social Psychology 85(2), 348-362 (2003) 
8. Gross, J.J., Levenson, R.W.: Hiding feelings: The acute effects of inhibiting negative and positive emotion. Journal of Abnormal Psychology 106, 95-103 (1997)

9. Winn, et al.: The Effect of Student Construction of Virtual Environments on the Performance of High- and Low-Ability Students. Annual Meeting of the American Educational Research Association (2003)

10. Pantelidis, V.: Reasons to use virtual reality in education. VR in the Schools 1(1) (1995)

11. Playmancer, http://www.playmancer.eu

12. Ben Moussa, M., Magnenat-Thalmann, N.: Applying affect recognition in serious games: The playMancer project. In: Egges, A., Geraerts, R., Overmars, M. (eds.) MIG 2009. LNCS, vol. 5884, pp. 53-62. Springer, Heidelberg (2009)

13. Replay, http: //www.replayproject.eu

14. Feldman, L.B., Gross, J.J., Conner, T., Benvenuto, M.: Knowing what you're feeling and knowing what to do about it: mapping the relation between emotion differentiation and emotion regulation. Cognition and Emotion 15, 713-724 (2001) 